\title{
On Wild Algebras and Super-Decomposable Pure-Injective Modules
}

\author{
Grzegorz Pastuszak ${ }^{1}[$
}

Received: 22 July 2021 / Accepted: 18 January 2022

(c) The Author(s) 2022

\begin{abstract}
Assume that $k$ is an algebraically closed field and $A$ is a finite-dimensional wild $k$-algebra. Recently, L. Gregory and M. Prest proved that in this case the width of the lattice of all pointed $A$-modules is undefined. Hence the result of $\mathrm{M}$. Ziegler implies that there exists a super-decomposable pure-injective $A$-module, if the base field $k$ is countable. Here we give a different and straightforward proof of this fact. Namely, we show that there exists a special family of pointed $A$-modules, called an independent pair of dense chains of pointed $A$ modules. This also yields the existence of a super-decomposable pure-injective $A$-module.
\end{abstract}

Keywords Wild algebras · Super-decomposable pure-injective modules · String algebras · Pointed modules

Mathematics Subject Classification 2010 Primary 16G20 · Secondary 03C60

\section{Introduction}

Tame and wild dichotomy of Yu. Drozd [2] states that the class of finite-dimensional algebras over algebraically closed fields divides into two disjoint classes: tame algebras and wild algebras. The class of wild algebras properly contains the class of strictly wild algebras. We refer the reader to [23] for definitions of these classes. Furthermore, A. Skowroński introduced in [24] a concept of the growth of a tame algebra. This yields a stratification of the class of tame algebras into domestic, linear and polynomial growth algebras. Tame algebras which are not of polynomial growth are called non-polynomial growth algebras.

Understanding various aspects of representation types is still one of the central topics of the representation theory of finite-dimensional algebras over algebraically closed fields. A good example supporting this fact is provided by the tame self-injective algebras. Indeed,

Presented by: Kenneth Goodearl

Dedicated to Professor Daniel Simson on the occasion of his 80th birthday

Grzegorz Pastuszak

past@mat.umk.pl

1 Faculty of Mathematics and Computer Science, Nicolaus Copernicus University, Chopina 12/18, 87-100 Toruń, Poland 
the representation theory of these algebras is well developed for the polynomial growth (see $[26,27])$, but much less is known for the non-polynomial growth. Recently, K. Erdmann and A. Skowroński introduced in [3] a prominent class of weighted surface algebras. These algebras are some special representation-infinite tame symmetric algebras (and hence selfinjective). Since most of them is of non-polynomial growth, they play a significant role in understanding the representation theory of all tame self-injective algebras.

The representation type is studied by using various concepts and methods. On the level of finite-dimensional modules we have, in particular, results on the shape of connected components of the Auslander-Reiten quiver or the component quiver, see for example [6, $25,29]$. On the level of infinite-dimensional modules, a fundamental characterization of the representation type is given in [1] in terms of generic modules. It is conjectured by M. Prest (see [18, Introduction]) that a finite-dimensional algebra $A$ over an algebraically closed field is of domestic representation type if and only if there is no super-decomposable pureinjective A-module (these modules do not have indecomposable direct summands, so they are of infinite dimension). This paper is related to the conjecture of Prest.

The problem of the existence of super-decomposable pure-injective $R$-modules is stated in [31] (for the concept of pure-injectivity we refer to [5, 11, 30] and [7, Chapter 7]). In this paper M. Ziegler proves in Lemma 7.8 (2) a fundamental criterion for such modules to exist, asserting that if the ring $R$ is countable, then $R$ possesses a super-decomposable pureinjective module if and only if the width of the lattice of all pp-formulas is undefined, see $[16$, Chapter 10] or $[17,7.3]$ for all the definitions. The later statement can be formulated in terms of the lattice of all pointed finitely-presented $R$-modules, see [22] and [9] for more details. We call the renowned result of Ziegler given in [31, Lemma 7.8 (2)] the Ziegler criterion.

The case when $R$ is a finite-dimensional algebra over a field $k$ is studied in many papers, see [10] for an up-to-date list of results in this direction and [14, Theorem 8.3] for the most recent one concerning self-injective algebras. Of course all the known results support the conjecture of Prest. The first result on the existence of super-decomposable pure-injective modules for finite-dimensional algebras is proved by M. Prest himself in [16, Theorem 13.7]. It states that these modules do exist over strictly wild algebras. For over 25 years it was not known whether this holds for wild algebras. Recently, L. Gregory and M. Prest prove in [4] that this is the case. Indeed, they show in [4, Theorem 2.1] that any representation embedding functor induces an embedding of lattices of pp-formulas. This implies that the width of the lattice of all pp-formulas over a wild algebra $A$ is undefined, and so there exists a super-decomposable pure-injective $A$-module, if the base field is countable (see Corollary 2.4 of [4]).

Recall that an independent pair of dense chains of pointed modules is some special family of pointed modules that allows to formulate a handy sufficient condition for the existence of a super-decomposable pure-injective module (see Section 2 for the details). This notion was introduced in [19] by G. Puninski (see also [22, Proposition 5.4], and especially [20, 21] for first applications to finite-dimensional algebras) and further generalized in [9]. It is successfully used in the series of papers $[8-10,15]$ which is devoted to the problem of the existence of super-decomposable pure-injective modules for strongly simply connected algebras, see [13, 28].

The present paper is devoted to show that if $A$ is a wild $k$-algebra over an algebraically closed field $k$, then there exists an independent pair of dense chains of pointed $A$-modules, see Theorems 3.1 and 3.2. The proof of this fact is rather straightforward and completely independent of [4]. 
We emphasize that Theorem 3.2 implies [4, Corollary 2.4] (see Theorem 2.4 for details), but the converse is not known. In this sense, Theorem 3.2 is stronger than [4, Corollary 2.4]. Regardless of this fact, our main result may be viewed as another and simple proof (accessible also to non-experts in model theory of modules) that wild algebras possess super-decomposable pure-injective modules. This shows a significant usefulness of independent pairs of dense chains of pointed modules once again.

The paper contains three sections. In Section 2 we collect basic information on pointed modules, recall the definition of an independent pair of dense chains of pointed modules and formulate the Ziegler criterion. We also introduce some special independent pairs of dense chains of pointed modules which we call strong. We recall from [9] that there are strong independent pairs of dense chains of pointed modules over string algebras of nonpolynomial growth, see Theorem 2.6. In Section 3 we present our main result. Indeed, Theorem 3.1 shows that representation embedding functors preserve strong independent pairs of dense chains of pointed modules. Then Theorem 3.2, stating the existence of an independent pair of dense chains of pointed modules and a super-decomposable pureinjective module for any wild algebra (if the base field is countable), is a direct consequence of Theorem 3.1 and Theorem 2.6. In the final remark we argue that considering strong independent pairs of dense chains and applying a non-trivial Theorem 2.6 is essential in the main results, see Remark 3.3.

Throughout, $k$ is a fixed algebraically closed field. By an algebra we mean a finitedimensional associative basic $k$-algebra with a unit. If $A$ is an algebra, then by an $A$-module we mean a left $A$-module. We denote by $A$-mod the category of all finitely-generated (hence finite-dimensional) left $A$-modules.

\section{The Lattice of Pointed Modules and a Sufficient Existence Condition}

In this section we recall some basic facts on pointed modules and related concepts. In particular, we present a sufficient condition for the existence of a super-decomposable pure-injective module in terms of independent pairs of dense chains of pointed modules.

Assume that $R$ is a ring with a unit. We denote by $R$-mod the category of all finitelypresented left $R$-modules. Assume that $\Theta \in R$-mod. A $\Theta$-pointed $R$-module is a pair $\left(M, \chi_{M}\right)$ where $M$ is a finitely-presented $R$-module and $\chi_{M}: \Theta \rightarrow M$ is an $R$-module homomorphism.

Assume that $\left(M, \chi_{M}\right)$ and $\left(N, \chi_{N}\right)$ are $\Theta$-pointed $R$-modules. Then a $\Theta$-pointed $R$ homomorphism from $\left(M, \chi_{M}\right)$ to $\left(N, \chi_{N}\right)$ is an $R$-homomorphism $f: M \rightarrow N$ such that $f \chi_{M}=\chi_{N}$. If $f: M \rightarrow N$ is a $\Theta$-pointed $R$-homomorphism from $\left(M, \chi_{M}\right)$ to $\left(N, \chi_{N}\right)$, we write $f:\left(M, \chi_{M}\right) \rightarrow\left(N, \chi_{N}\right)$. If $f: M \rightarrow N$ is an isomorphism, we call $f:\left(M, \chi_{M}\right) \rightarrow\left(N, \chi_{N}\right)$ a $\Theta$-pointed isomorphism and the corresponding $\Theta$-pointed modules $\left(M, \chi_{M}\right)$ and $\left(N, \chi_{N}\right) \Theta$-isomorphic.

Assume that $t \in \mathbb{N}, t \geq 1, \Theta=R^{t}$ and $\left(M, \chi_{M}\right)$ is a $\Theta$-pointed $R$-module. Assume that $e_{1}, \ldots, e_{t}$ form the $R$-base of the module $\Theta$. The homomorphism $\chi_{M}$ is uniquely determined by the elements $\chi\left(e_{1}\right), \ldots, \chi\left(e_{t}\right) \in M$. This yields that any $\Theta$-pointed $R$-module can be identified with a tuple $\left(M, m_{1}, \ldots, m_{t}\right)$ where $M$ is an $R$-module and $m_{1}, \ldots, m_{t} \in M$. Moreover, a $\Theta$-pointed $R$-homomorphism from $\left(M, m_{1}, \ldots, m_{t}\right)$ to $\left(N, n_{1}, \ldots, n_{t}\right)$ can be identified with an $R$-homomorphism $f: M \rightarrow N$ such that $f\left(m_{i}\right)=n_{i}$, for $i=1, \ldots, t$. In case $\Theta=R$, we simply speak about pointed modules and pointed homomorphisms. 
Let $P_{R}^{\Theta}$ be the set of all $\Theta$-isomorphism classes of $\Theta$-pointed $R$-modules. Let $\equiv$ be a binary relation on $P_{R}^{\Theta}$ defined by $\left(M, \chi_{M}\right) \equiv\left(N, \chi_{N}\right)$ if and only if there exist two pointed homomorphisms $f:\left(M, \chi_{M}\right) \rightarrow\left(N, \chi_{N}\right)$ and $g:\left(N, \chi_{N}\right) \rightarrow\left(M, \chi_{M}\right)$. Then $\equiv$ is an equivalence relation and the quotient set $\mathcal{P}_{R}^{\Theta}=P_{R}^{\Theta} / \equiv$ is a poset with respect to the relation $\leq$ defined by $\overline{\left(M, \chi_{M}\right)} \leq \overline{\left(N, \chi_{N}\right)}$ if and only if there exists a pointed homomorphism $f:\left(N, \chi_{N}\right) \rightarrow\left(M, \chi_{M}\right)$. We denote by $\overline{\left(S, \chi_{S}\right)}$ the $\equiv$-class of a $\Theta$-pointed $R$-module $(S, \chi S)$.

The poset $\mathcal{P}_{R}^{\Theta}$ is a modular lattice with respect to the operations $\oplus$ and $*$ defined below, see [16, Chapter 10] for details.

Assume that $\left(M, \chi_{M}\right),\left(N, \chi_{N}\right)$ are $\Theta$-pointed $R$-modules. A $\Theta$-pointed $R$-module $(M \oplus$ $\left.N, \chi_{M \oplus N}\right)$ where $\chi_{M \oplus N}(l)=\left(\chi_{M}(l), \chi_{N}(l)\right)$ for any $l \in \Theta$ is the pointed direct sum of $\left(M, \chi_{M}\right)$ and $\left(N, \chi_{N}\right)$. We set $\left(M, \chi_{M}\right) \oplus\left(N, \chi_{N}\right)=\left(M \oplus N, \chi_{M \oplus N}\right)$.

Assume that $M * N$ is the pushout of $\chi_{M}$ and $\chi_{N}$, that is,

$$
M * N=M \oplus N /\left\{\left(\chi_{M}(l),-\chi_{N}(l)\right) ; l \in \Theta\right\} .
$$

Moreover, let $\epsilon_{M}: M \rightarrow M * N, \epsilon_{N}: N \rightarrow M * N$ be the $R$-module homomorphisms given by $\epsilon_{M}(m)=\overline{(m, 0)}, \epsilon_{N}(n)=\overline{(0, n)}$ for any $m \in M, n \in N$. A $\Theta$-pointed $R$-module $\left(M * N, \chi_{M * N}\right)$ where $\chi_{M * N}=\epsilon_{M} \chi_{M}=\epsilon_{N} \chi_{N}$ is the pointed pushout of $\left(M, \chi_{M}\right)$ and $\left(N, \chi_{N}\right)$. We set $\left(M, \chi_{M}\right) *\left(N, \chi_{N}\right)=\left(M * N, \chi_{M * N}\right)$. Recall that

$$
\begin{aligned}
& \sup \left\{\overline{\left(M, \chi_{M}\right)}, \overline{\left(N, \chi_{N}\right)}\right\}=\overline{\left(M \oplus N, \chi_{M \oplus N}\right)}, \\
& \inf \left\{\overline{\left(M, \chi_{M}\right)}, \overline{\left(N, \chi_{N}\right)}\right\}=\overline{\left(M * N, \chi_{M * N}\right),}
\end{aligned}
$$

see [9] for details. It is worth to mention that if $\Theta=R^{t}$, then the lattice $\mathcal{P}_{R}^{\Theta}$ is equivalent to the lattice of all pp-formulas with $t$ free variables $(t \geq 1)$, see [16, Chapter 10] and [17, 7.2].

We recall definitions of wide lattices of pointed modules and independent pairs of dense chains of pointed modules. Moreover, we present in Theorem 2.3 the relation between these notions.

We say that a lattice $\mathcal{L} \subseteq \mathcal{P}_{R}^{\Theta}$ of $\Theta$-pointed $R$-modules is wide if and only if for any $\overline{\left(M_{p}, \chi_{M_{p}}\right)}<\overline{\left(M_{q}, \chi_{M_{q}}\right)} \in \mathcal{L}$ there are incomparable elements $\overline{\left(M, \chi_{M}\right)}, \overline{\left(N, \chi_{N}\right)}$ of $\mathcal{L}$ such that

$$
\begin{gathered}
\overline{\left(M_{p}, \chi_{M_{p}}\right)}<\overline{\left(M, \chi_{M}\right)}, \overline{\left(N, \chi_{N}\right)}<\overline{\left(M_{q}, \chi_{M_{q}}\right)}, \\
\overline{\left(M_{p}, \chi_{M_{p}}\right)} \leq \overline{\left(M * N, \chi_{M * N}\right)}<\overline{\left(M \oplus N, \chi_{M \oplus N}\right)} \leq \overline{\left(M_{q}, \chi_{M_{q}}\right)} .
\end{gathered}
$$

In case the lattice $\mathcal{P}_{R}^{\Theta}$ contains a wide sublattice $\mathcal{L}$, we say that the width of $\mathcal{P}_{R}^{\Theta}$ is undefined. The above definition is a special case of a general definition of a wide lattice, see for example Section 3 of [9].

Assume that $C$ is a set. A family $\left\{\left(M_{q}, \chi_{M_{q}}\right) ; q \in C\right\}$ of $\Theta$-pointed $R$-modules is denoted by $\left(M_{q}, \chi_{M_{q}}\right)_{q \in C}$. Let $\mathbb{Q}$ be the set of rational numbers viewed as a poset with respect to the natural ordering $\leq$. Recall that a poset $P$ is a $\mathbb{Q}$-chain if and only if it is a dense chain without end points. It is well known that any $\mathbb{Q}$-chain is isomorphic as a poset with the set $\mathbb{Q}$.

Assume $R$ is a ring with a unit and $\Theta$ is a finitely-presented $R$-module. The following definitions were introduced in [22] and generalized in [9].

Definition 2.1 Assume that $C$ is a $\mathbb{Q}$-chain. A dense chain of $\Theta$-pointed $R$-modules is a family $\left(M_{q}, \chi_{M_{q}}\right)_{q \in C}$ of $\Theta$-pointed $R$-modules such that:

(a) the ring $\operatorname{End}_{R}\left(M_{q}\right)$ is local and $\chi_{M_{q}} \neq 0$, for any $q \in C$, 
(b) there exist $\Theta$-pointed homomorphisms

$$
\mu_{q, q^{\prime}}:\left(M_{q}, \chi_{M_{q}}\right) \rightarrow\left(M_{q^{\prime}}, \chi_{M_{q^{\prime}}}\right)
$$

for any $q<q^{\prime} \in C$,

(c) the pointed modules $\left(M_{q}, \chi_{M_{q}}\right)$ and $\left(M_{q^{\prime}}, \chi_{M_{q^{\prime}}}\right)$ are not $\Theta$-isomorphic, for any $q \neq$ $q^{\prime} \in C$.

Definition 2.2 An independent pair of dense chains of $\Theta$-pointed $R$-modules is a pair $\left(\left(M_{q}, \chi_{M_{q}}\right)_{q \in C_{1}},\left(N_{t}, \chi_{N_{t}}\right)_{t \in C_{2}}\right)$ of dense chains of $\Theta$-pointed $R$-modules such that:

(a) the endomorphism ring $\operatorname{End}_{R}\left(M_{q} * N_{t}\right)$ is local, for any $q \in C_{1}, t \in C_{2}$, where $\left(M_{q} *\right.$ $\left.N_{t}, \chi_{M_{q} * N_{t}}\right)=\left(M_{q}, \chi_{M_{q}}\right) *\left(N_{t}, \chi_{N_{t}}\right)$,

(b) the pointed module $\left(M_{q}, \chi_{M_{q}}\right) *\left(N_{t}, \chi_{N_{t}}\right)$ is not $\Theta$-isomorphic to $\left(M_{q^{\prime}}, \chi_{M_{q^{\prime}}}\right) *$ $\left(N_{t}, \chi_{N_{t}}\right)$ nor to $\left(M_{q}, \chi_{M_{q}}\right) *\left(N_{t^{\prime}}, \chi_{N_{t^{\prime}}}\right)$, for any $q \neq q^{\prime} \in C_{1}, t \neq t^{\prime} \in C_{2}$.

Independent pairs of dense chains of pointed modules generate wide lattices of pointed modules in the following way.

Theorem 2.3 Assume that the pair $\left(\left(M_{q}, \chi_{M_{q}}\right)_{q \in C_{1}},\left(N_{t}, \chi_{N_{t}}\right)_{t \in C_{2}}\right)$ is an independent pair of dense chains of $\Theta$-pointed $R$-modules. Then the lattice

$$
\operatorname{Gen}\left({\overline{\left(M_{q}, \chi_{M_{q}}\right)}}_{q \in C_{1}} \cup{\overline{\left(N_{t}, \chi_{N_{t}}\right)}}_{t \in C_{2}}\right) \text {, }
$$

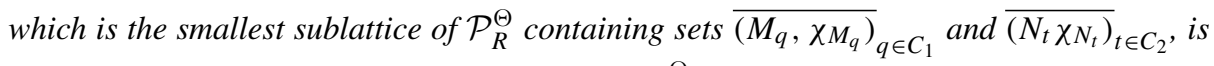
a wide lattice. Therefore the width of the lattice $\mathcal{P}_{R}^{\Theta}$ is undefined.

Proof The assertion is a direct consequence of [9, Theorem 3.4].

It is not known whether the existence of a wide sublattice of $\mathcal{P}_{R}^{\Theta}$ (or, equivalently, a wide sublattice of the lattice of all pp-formulas over $R$ ) implies the existence of an independent pair of dense chains of $\Theta$-pointed $R$-modules. However, this is rather unexpected, since the latter condition implies that $\mathcal{P}_{R}^{\Theta}$ contains a lattice freely generated by two chains, see [20], which seems to be a special situation.

The assertion (1) of the following theorem is the Ziegler's criterion, see [31], and (2) is a handy version of this criterion. Observe that (2) follows directly from (1) and Theorem 2.3.

Theorem 2.4 Assume that $R$ is a countable ring with a unit and $\Theta$ is a finitely presented $R$-module.

1. If the lattice $\mathcal{P}_{R}^{\Theta}$ of $\Theta$-pointed $R$-modules has width undefined, then there exists a superdecomposable pure-injective $R$-module.

2. If there is an independent pair of dense chains of $\Theta$-pointed $R$-modules, then there is a super-decomposable pure-injective $R$-module.

We apply the above theorem only when $R$ is a finite-dimensional $k$-algebra. Note that in this case any finitely-presented $R$-module $M$ is finite-dimensional and $\operatorname{End}_{R}(M)$ is local if and only if $M$ is indecomposable. Moreover, it is easy to see that $R$ is countable if and only if the field $k$ is countable. 
Assume that $\left(M, \chi_{M}\right)$ and $\left(N, \chi_{N}\right)$ are $\Theta$-pointed $R$-modules. Note that if we have $\left(M, \chi_{M}\right) \cong\left(N, \chi_{N}\right)$, then $M \cong N$, but the converse does not hold in general. This justifies the following special version of Definitions 2.1 and 2.2.

Definition 2.5 Assume that $R$ is a ring with a unit.

1. A dense chain $\left(M_{q}, \chi_{M_{q}}\right)_{q \in C}$ of $\Theta$-pointed $R$-modules is strong if and only if $M_{q}$ and $M_{q^{\prime}}$ are not isomorphic (as $R$-modules), for any $q<q^{\prime} \in C$.

2. An independent pair $\left(\left(M_{q}, \chi_{M_{q}}\right)_{q \in C_{1}},\left(N_{t}, \chi_{N_{t}}\right)_{t \in C_{2}}\right)$ of dense chains of $\Theta$-pointed $R$ modules is strong if and only if dense chains $\left(M_{q}, \chi_{M_{q}}\right)_{q \in C_{1}}$ and $\left(N_{t}, \chi_{N_{t}}\right)_{t \in C_{2}}$ are strong and the module $M_{q} * N_{t}$ is not isomorphic (as an $R$-module) to $M_{q^{\prime}} * N_{t}$ nor to $M_{q} * N_{t^{\prime}}$, for any $q \neq q^{\prime} \in C_{1}, t \neq t^{\prime} \in C_{2}$.

In Section 3 we apply the following theorem, see [8, Theorem 5.7].

Theorem 2.6 Assume that $A$ is a string algebra of non-polynomial growth. There exists a strong independent pair of dense chains of pointed modules in A-mod.

The above theorem is a non-trivial result with important consequences. Namely, it allows to prove that there are independent pairs of dense chains of pointed modules (and hence super-decomposable pure-injective modules in the case of countable base fields) over all pg-critical algebras, strongly simply connected algebras of non-polynomial growth and nonpolynomial growth algebras having strongly simply connected Galois coverings (see [8-10] for the proofs and $[13,28]$ for the definitions).

\section{The Main Result}

This section is devoted to prove the main result of the paper. In Theorem 3.1 we show that representation embeddings preserve strong independent pairs of dense chains of pointed modules. It follows directly from Theorem 3.1 and Theorem 2.6 that any wild algebra $A$ possesses an independent pair of dense chains of pointed modules. Hence there exists a super-decomposable pure-injective $A$-module, if the base field is countable. These facts are stated in Theorem 3.2.

Throughout the section, $A, B$ are $k$-algebras. All functors considered are covariant functors. Assume that $F: B$-mod $\rightarrow A$-mod is a functor and $\left(M, \chi_{M}\right)$ is a $\Theta$-pointed $B$-module, for some $\Theta \in B$-mod. The $F(\Theta)$-pointed $A$-module $\left(F(M), F\left(\chi_{M}\right)\right)$ is denoted by $F\left(M, \chi_{M}\right)$. If $\left(M_{q}, \chi_{M_{q}}\right)_{q \in C}$ is a family of $\Theta$-pointed $B$-modules, then $F\left(M_{q}, \chi_{M_{q}}\right)_{q \in C}$ denotes the family $\left(F\left(M_{q}\right), F\left(\chi_{M_{q}}\right)\right)_{q \in C}$ of $F(\Theta)$-pointed $A$-modules.

Recall that a functor $F: B$-mod $\rightarrow A$-mod is a representation embedding if and only if $F$ is exact, respects the isomorphism classes (that is, $F(X) \cong F(Y)$ implies $X \cong Y$, for any $B$-modules $X, Y$ ) and carries indecomposable modules to indecomposable ones. These conditions yield representation embeddings are faithful functors.

Theorem 3.1 Assume that $A, B$ are $k$-algebras and $F: B$-mod $\rightarrow A$-mod is a representation embedding. Assume that $\left(\left(M_{q}, \chi_{M_{q}}\right)_{q \in C_{1}},\left(N_{t}, \chi_{N_{t}}\right)_{t \in C_{2}}\right)$ is a strong independent pair of dense chains of $\Theta$-pointed $B$-modules. Then

$$
\left(F\left(M_{q}, \chi_{M_{q}}\right)_{q \in C_{1}}, F\left(N_{t}, \chi_{N_{t}}\right)_{t \in C_{2}}\right)
$$


is an independent pair of dense chains of $F(\Theta)$-pointed A-modules.

Proof We show that $F\left(M_{q}, \chi_{M_{q}}\right)_{q \in C_{1}}$ is a dense chain of $F(\Theta)$-pointed $A$-modules (similar arguments show that $F\left(N_{t}, \chi_{N_{t}}\right)_{t \in C_{2}}$ is a dense chain of $F(\Theta)$-pointed $A$-modules as well). Indeed, assume that $q \in C_{1}$. The module $F\left(M_{q}\right)$ is indecomposable, because $M_{q}$ is indecomposable. Since $F$ is a faithful functor, we get $F\left(\chi_{M_{q}}\right) \neq 0$, because $\chi_{M_{q}} \neq 0$.

Assume that $q<q^{\prime}$ and $\mu_{q, q^{\prime}}:\left(M_{q}, \chi_{M_{q}}\right) \rightarrow\left(M_{q^{\prime}}, \chi_{M_{q^{\prime}}}\right)$ is a $\Theta$-pointed homomorphism. Since $\mu_{q, q^{\prime}} \chi_{M_{q}}=\chi_{M_{q^{\prime}}}$, we get $F\left(\mu_{q, q^{\prime}}\right) F\left(\chi_{M_{q}}\right)=F\left(\chi_{M_{q^{\prime}}}\right)$, so $F\left(\mu_{q, q^{\prime}}\right)$ is a $F(\Theta)$-pointed homomorphism from $F\left(M_{q}, \chi_{M_{q}}\right)$ to $F\left(M_{q^{\prime}}, \chi_{M_{q^{\prime}}}\right)$.

Furthermore, $F(\Theta)$-pointed modules $F\left(M_{q}, \chi_{M_{q}}\right)$ and $F\left(M_{q^{\prime}}, \chi_{M_{q^{\prime}}}\right)$ are not isomorphic, for any $q \neq q^{\prime}$. Indeed, we have $F\left(M_{q}\right) ¥ F\left(M_{q^{\prime}}\right)$, because $M_{q} ¥ M_{q^{\prime}}$. This shows that $F\left(M_{q}, \chi_{M_{q}}\right)_{q \in C_{1}}$ is a dense chain of $F(\Theta)$-pointed $A$-modules.

We show that that dense chains $F\left(M_{q}, \chi_{M_{q}}\right)_{q \in C_{1}}$ and $F\left(N_{t}, \chi_{N_{t}}\right)_{t \in C_{2}}$ form an independent pair. Indeed, the module $F\left(M_{q} * N_{t}\right)$ is indecomposable, because $M_{q} * N_{t}$ is indecomposable, for any $q \in C_{1}, t \in C_{2}$.

Observe that the functor $F: \bmod (B) \rightarrow \bmod (A)$ preserves finite colimits (and finite limits), since it is exact, see [12, VIII.3]. This implies that

$$
F\left(\left(M_{q}, \chi_{M_{q}}\right) *\left(N_{t}, \chi_{N_{t}}\right)\right) \cong F\left(M_{q}, \chi_{M_{q}}\right) * F\left(N_{t}, \chi_{N_{t}}\right),
$$

for any $q \in C_{1}, t \in C_{2}$. In particular, we get $F\left(M_{q} * N_{1}\right) \cong F\left(M_{q}\right) * F\left(N_{t}\right)$.

Assume that $F\left(M_{q}, \chi_{M_{q}}\right) * F\left(N_{t}, \chi_{N_{t}}\right)$ is isomorphic with $F\left(M_{q}, \chi_{M_{q}}\right) * F\left(N_{t^{\prime}}, \chi_{N_{t^{\prime}}}\right)$, for some $q \in C_{1}$ and $t \neq t^{\prime} \in C_{2}$. Then we get

$$
F\left(M_{q} * N_{t}\right) \cong F\left(M_{q}\right) * F\left(N_{t}\right) \cong F\left(M_{q}\right) * F\left(N_{t^{\prime}}\right) \cong F\left(M_{q} * N_{t^{\prime}}\right),
$$

which yields $M_{q} * N_{t} \cong M_{q} * N_{t^{\prime}}$. Since this is not the case, we get that $F\left(M_{q}, \chi_{M_{q}}\right) *$ $F\left(N_{t}, \chi_{N_{t}}\right)$ is not isomorphic with $F\left(M_{q}, \chi_{M_{q}}\right) * F\left(N_{t^{\prime}}, \chi_{N_{t^{\prime}}}\right)$. Similar arguments show that $F\left(M_{q}, \chi_{M_{q}}\right) * F\left(N_{t}, \chi_{N_{t}}\right)$ is not isomorphic with $F\left(M_{q^{\prime}}, \chi_{M_{q^{\prime}}}\right) * F\left(N_{t}, \chi_{N_{t}}\right)$ as well, for any $q^{\prime} \neq q \in C_{1}$. This shows the assertion.

An algebra $A$ is of wild representation type (or wild) if and only if there exists a representation embedding functor $F: C$-mod $\rightarrow A$-mod, for any $k$-algebra $C$.

Theorem 3.2 Assume that $A$ is a wild $k$-algebra over an algebraically closed field $k$. There exists an independent pair of dense chains of $\Xi$-pointed modules, for some A-module $\Xi$. Therefore there exists a super-decomposable pure-injective A-module, if the base field $k$ is countable.

Proof Since $A$ is a wild algebra, there exists a representation embedding functor $F$ : $\Lambda$-mod $\rightarrow A$-mod where $\Lambda$ is the string algebra considered in Theorem 2.6. Thus the assertion follows from Theorem 3.1 and Ziegler's criterion (Theorem 2.4 (2)).

Remark 3.3 We emphasize it should not be expected that a representation embedding functor $F$ preserves arbitrary dense chains of pointed modules. Observe that if we have $\left(F\left(M_{q}\right), F\left(\chi_{M_{q}}\right)\right) \cong\left(F\left(M_{q^{\prime}}\right), F\left(\chi_{M_{q^{\prime}}}\right)\right)$, then $F\left(M_{q}\right) \cong F\left(M_{q^{\prime}}\right)$ and hence $M_{q} \cong M_{q^{\prime}}$, but in general $\left(M_{q}, \chi_{M_{q}}\right)$ may not be isomorphic to $\left(M_{q^{\prime}}, \chi_{M_{q^{\prime}}}\right)$ as pointed modules. Indeed, we only know that the local algebra $\operatorname{End}_{B}\left(M_{q}\right)$ is a subalgebra of the local algebra $\operatorname{End}_{A}\left(F\left(M_{q}\right)\right.$ ), but the latter may be much larger (we identify the algebra $\operatorname{End}_{B}\left(M_{q}\right)$ with its image under the functor $F: B$-mod $\rightarrow A$-mod). 
To be more concrete, observe that $\left(M_{q}, \chi_{M_{q}}\right)$ is isomorphic to $\left(M_{q^{\prime}}, \chi_{M_{q^{\prime}}}\right.$ ) (as pointed modules) if and only if $\left(F\left(M_{q}\right), F\left(\chi_{M_{q}}\right)\right)$ is isomorphic to the pointed module $\left(F\left(M_{q^{\prime}}\right), F\left(\chi_{M_{q^{\prime}}}\right)\right)$ with a pointed isomorphism $\alpha: F\left(M_{q}\right) \rightarrow F\left(M_{q^{\prime}}\right)$ of the form $F(f)$, for some $f: M_{q} \rightarrow M_{q^{\prime}}$ (this follows easily from the fact that $F$ is faithful). Since representation embeddings are not full in general, it seems natural to think it may happen that all the pointed isomorphisms $\alpha:\left(F\left(M_{q}\right), F\left(\chi_{M_{q}}\right)\right) \rightarrow\left(F\left(M_{q^{\prime}}\right), F\left(\chi_{M_{q^{\prime}}}\right)\right)$ belong to the set $\operatorname{End}_{A}\left(F\left(M_{q}\right)\right) \backslash \operatorname{End}_{B}\left(M_{q}\right)$.

Let us recall that all the known and natural examples of independent pairs of dense chains of pointed modules for finite dimensional algebras, coming mainly from the papers [8-10], are strong. Hence finding an example of a representation embedding which does not preserve arbitrary dense chains of pointed modules may be a hard open problem.

The above discussion shows that it is essential to consider strong independent pairs of dense chains of pointed modules in Theorem 3.1 and hence applying Theorem 2.6 is crucial.

Declarations The authors did not receive support from any organization for the submitted work. The authors have no relevant financial or non-financial interests to disclose. Data sharing not applicable to this article as no datasets were generated or analysed during the current study.

Open Access This article is licensed under a Creative Commons Attribution 4.0 International License, which permits use, sharing, adaptation, distribution and reproduction in any medium or format, as long as you give appropriate credit to the original author(s) and the source, provide a link to the Creative Commons licence, and indicate if changes were made. The images or other third party material in this article are included in the article's Creative Commons licence, unless indicated otherwise in a credit line to the material. If material is not included in the article's Creative Commons licence and your intended use is not permitted by statutory regulation or exceeds the permitted use, you will need to obtain permission directly from the copyright holder. To view a copy of this licence, visit http://creativecommons.org/licenses/by/4.0/.

\section{References}

1. Crawley-Boevey, W.W.: Tame algebras and modules generic. Proc. London Math. Soc. 63, 241-264 (1991)

2. Drozd, Yu.A.: Tame and wild matrix problems. In: Representations and Quadratic Forms, Kiev, 39-74 (in Russian) (1979)

3. Erdmann, K., Skowroński, A.: Weighted surface algebras. J. Algebra 505, 490-558 (2018)

4. Gregory, L., Prest, M.: Representation embeddings, interpretation functors and controlled wild algebras. J. Lond. Math. Soc. (2) 94(3), 747-766 (2016)

5. Huisgen-Zimmermann, B.: Purity, Algebraic Compactness, Direct Sum Decompositions, and Representation Type. In: Infinite Length Modules (Bielefeld, 1998), pp. 331-367. Birkhäuser, Basel, Trends Math. (2000)

6. Jaworska, A., Skowroński, A.: The component quiver of a self-injective Artin algebra. Colloq. Math 122(2), 233-239 (2011)

7. Jensen, Ch.U.., Lenzing, H.: Model Theoretic Algebra with particular emphasis on Fields, Rings, Modules. Algebra, Logic and Applications, vol. 2. Gordon and Breach, New York (1989)

8. Kasjan, S., Pastuszak, G.: On two tame algebras with super-decomposable pure-injective modules. Colloq. Math. 123, 249-276 (2011)

9. Kasjan, S., Pastuszak, G.: On the existence of super-decomposable pure-injective modules over strongly simply connected algebras of non-polynomial growth. Colloq. Math. 136, 179-220 (2014)

10. Kasjan, S., Pastuszak, G.: Super-decomposable pure-injective modules over algebras with strongly simply connected Galois coverings. J. Pure Appl. Algebra 220(8), 2985-2999 (2016)

11. Kiełpiński, R.: On $\Gamma$-pure-injective modules. Bull. Acad. Polon. Sci. Sér. Sci. Math. Astronom. Phys. 15, 127-131 (1967)

12. Mac Lane, S.: Categories for the working mathematician graduate texts in mathematics, vol. 5. SpringerVerlag, New York (1998) 
13. Nörenberg, R., Skowroński, A.: Tame minimal non-polynomial growth simply connected algebras. Colloq. Math. 73, 301-330 (1997)

14. Pastuszak, G.: On Krull-Gabriel dimension and Galois coverings. Adv. Math. 349, 959-991 (2019)

15. Pastuszak, G.: Strongly simply connected algebras with super-decomposable pure-injective modules. J. Pure Appl. Algebra 219(8), 3314-3321 (2015)

16. Prest, M.: Model theory and modules. London Mathematical Society Lecture Note Series, vol. 130. Cambridge University Press, Cambridge (1988)

17. Prest, M.: Purity, spectra and localization Encyclopedia of mathematics and its applications, vol. 121. Cambridge University Press, Cambridge (2009)

18. Prest, M.: Superdecomposable pure-injective modules. In: Advances in Representation Theory of Algebras, EMS Series of Congress Reports 263-296. (2013)

19. Puninski, G.: When a super-decomposable pure-injective module over a serial ring exists. Rings, modules, algebras, and abelian groups, 449-463, Lecture Notes in Pure and Appl Math., vol. 236. Dekker, New York (2004)

20. Puninski, G.: How to construct a 'concrete' superdecomposable pure-injective module over a string algebra. J. Pure Appl. Algebra 212, 704-717 (2008)

21. Puninski, G.: Superdecomposable pure-injective modules exist over some string algebras. Proc. Amer. Math. Soc. 132, 1891-1898 (2004)

22. Puninski, G., Puninskaya, V., Toffalori, C.: Krull-Gabriel dimension and the model-theoretic complexity of the category of modules over group rings of finite groups. J. Lond. Math. Soc. 78, 125-142 (2008)

23. Simson, D., Skowroński, A.: Elements of the representation theory of associative algebras 3: Representation-Infinite Tilted Algebras, London Mathematical Society Student Texts 72, Cambridge University Press (2007)

24. Skowroński, A.: Algebras of Polynomial Growth. Topics in Algebra, Banach Center Publ. 26, Part, vol. 1, pp. 535-568. PWN, Warsaw (1990)

25. Skowroński, A.: Module categories over tame algebras, in: Representations Theory of Algebras ad Related Topics. CMS Conf. Proc. 19, 218-313 (1996)

26. Skowroński, A.: Selfinjective algebras of polynomial growth. Math. Ann. 285, 177-199 (1989)

27. Skowroński, A.: Selfinjective algebras: finite and tame type. Contemp. Math. 406, 169-238 (2006)

28. Skowroński, A.: Simply connected algebras Hochschild cohomologies in: Representations of Algebras. CMS Conf. Proc. 14, 431-447 (1993)

29. Skowroński, A.: Tame module categories of finite dimensional algebras, in: Trends in Ring Theory. CMS Conf. Proc. 22, 187-219 (1998)

30. Stenström, B., Pure submodules: Ark. Mat.,7, 159-171 (1967)

31. Ziegler, M.: Model theory of modules. Annals of Pure and Applied Logic 26, 149-213 (1984)

Publisher's Note Springer Nature remains neutral with regard to jurisdictional claims in published maps and institutional affiliations. 